University of Nebraska - Lincoln

DigitalCommons@University of Nebraska - Lincoln

Faculty Publications from the Department of Electrical \& Computer Engineering, Department Electrical and Computer Engineering

7-1-1998

\title{
In situ infrared and visible-light ellipsometric investigations of boron nitride thin films at elevated temperatures
}

\author{
E. Franke \\ Mathias Schubert \\ University of Nebraska-Lincoln, mschubert4@unl.edu \\ J.-D. Hecht \\ University of Nebraska-Lincoln \\ H. Neumann \\ Institute of Surface Modification, D-04303 Leipzig, Germany \\ T. E. Tiwald \\ University of Nebraska-Lincoln \\ See next page for additional authors
}

Institute of Surface Modification, D-04303 Leipzig, Germany, efranke3@unl.edu

Follow this and additional works at: https://digitalcommons.unl.edu/electricalengineeringfacpub

Part of the Electrical and Computer Engineering Commons

Franke, E.; Schubert, Mathias; Hecht, J.-D.; Neumann, H.; Tiwald, T. E.; Thompson, Daniel W.; Yao, H.; and Woollam, John A., "In situ infrared and visible-light ellipsometric investigations of boron nitride thin films at elevated temperatures" (1998). Faculty Publications from the Department of Electrical and Computer Engineering. 14.

https://digitalcommons.unl.edu/electricalengineeringfacpub/14

This Article is brought to you for free and open access by the Electrical \& Computer Engineering, Department of at DigitalCommons@University of Nebraska - Lincoln. It has been accepted for inclusion in Faculty Publications from the Department of Electrical and Computer Engineering by an authorized administrator of DigitalCommons@University of Nebraska - Lincoln. 
Authors

E. Franke, Mathias Schubert, J.-D. Hecht, H. Neumann, T. E. Tiwald, Daniel W. Thompson, H. Yao, and John A. Woollam 


\title{
In situ infrared and visible-light ellipsometric investigations of boron nitride thin films at elevated temperatures
}

\author{
E. Franke, M. Schubert, J.-D. Hecht, and H. Neumann \\ Institute of Surface Modification, D-04303 Leipzig, Germany \\ T. E. Tiwald, D. W. Thompson, H. Yao, and J. A. Woollama) \\ Center for Microelectronic and Optical Materials Research and Department of Electrical Engineering, \\ University of Nebraska, Lincoln, Nebraska 68588 \\ J. Hahn \\ Institute of Physics, Faculty of Science, Technical University Chemnitz, D-09107 Chemnitz, Germany
}

(Received 16 February 1998; accepted for publication 3 April 1998)

\begin{abstract}
In situ infrared (IR) spectroscopy and visible-light (VIS) spectroscopic ellipsometry over the spectral range from 700 to $2000 \mathrm{~cm}^{-1}$ and $1.5-3.5 \mathrm{eV}$, respectively, were used to investigate the optical behavior of boron nitride $(\mathrm{BN})$ thin films at temperatures from room temperature $(\mathrm{RT})$ to $600{ }^{\circ} \mathrm{C}$. The polycrystalline hexagonal $(\mathrm{h})$ and mixed-phase $\mathrm{h}-$ and cubic $(c)$-BN thin films were deposited by magnetron sputtering on [001] silicon. We observe a reversible moisture incorporation process in as-grown h-BN samples. When stored in normal ambient, the h-BN thin films absorb water into thin-film micropores. When annealed in ultrahigh vacuum or a dry nitrogen atmosphere, the samples expel moisture but retain their microstructure. This is observable by reduction of the thin-film refractive indices in accordance with changes in the IR lattice resonance behavior. The optical properties of high $c$-BN content thin films remain unchanged during annealing. And both intrinsic h- and $c$-BN thin-film VIS refractive indices are nearly temperature independent, at least up to $600{ }^{\circ} \mathrm{C}$. Therefore, RT BN optical constants can be used for feedback loop control in in situ thin-film growth at temperatures up to $600{ }^{\circ} \mathrm{C}$. [S0021-8979(98)07613-0]
\end{abstract}

\section{INTRODUCTION}

Because of the superior mechanical and optical material properties of hexagonal (h) and cubic (c) boron nitride $(\mathrm{BN})$, thin-film deposition of $\mathrm{BN}$ has recently gained wide interest. Various chemical vapor deposition (CVD) and physical vapor-phase (PVD) deposition techniques, such as plasmaenhanced CVD, ${ }^{1,2}$ magnetron sputtering, ${ }^{3}$, pulsed-laser deposition, ${ }^{4}$ ion-beam assisted deposition, ${ }^{5,6}$ or reactive evaporation, ${ }^{7}$ have been successfully used for deposition of $\mathrm{BN}$ thin films. Extensive work was reported on modification of the deposition method and selection of optimum-growth conditions for the preparation of high-quality $c$-BN thin films. However, most of the PVD and CVD deposition processes yield polycrystalline $\mathrm{BN}$ material with a porous film structure. The volume fraction of the micropores depends on various deposition parameters, such as the substrate temperature, ion energies, or ion-flux densities. As a result, thin-film aging effects are mainly caused by absorption of water molecules due to the thin-film microporosity followed by a BN hydrolysis as was pointed out by Hubácek and Ueki. ${ }^{8}$

Infrared (IR) $)^{4,9-14}$ and Raman spectroscopy, ${ }^{15-20}$ as well as IR and visible-light (VIS) spectroscopic ellipsometry $(\mathrm{SE})^{21-25}$ were used to investigate BN thin-film optical and structural properties. It was demonstrated that polarized IR reflection (PIRR) measurements, ${ }^{26,27}$ IR-SE ${ }^{28-34}$ and VIS-SE ${ }^{25,30}$ are useful techniques to resolve the BN micro-

\footnotetext{
a)Electronic mail: jwoollam@unl.edu
}

structure from the thin-film optical properties.

In situ SE has recently gained appreciable interest for thin-film growth control for various deposition techniques (see, e.g., Collins et al., Ref. 31). However, due to elevated growth temperatures, the materials involved may alter their optical properties which has to be taken into consideration for feedback loop control of, e.g., BN thin-film deposition by ion-beam assisted techniques. We therefore turn our attention in this article to optical studies of as-grown BN samples at elevated temperatures.

Ellipsometry is known as a precise and nondestructive optical method which determines both phase and absolute value of the thin-film reflectance ratio $\rho$, defined as $\rho$ $=R_{p} / R_{s}=\tan \Psi \exp (i \Delta)$, where $R_{p}$ and $R_{s}$ are the reflection coefficients for light polarization parallel $(p)$, and perpendicular $(s)$ to the plane of incidence, respectively. ${ }^{32}$ The ellipsometric parameters $\Psi$ and $\Delta$ therefore contain information for both $p$ - and $s$-polarized light components reflected from the sample surface. In order to analyze the ellipsometric data, one needs to model the sample structure as well as the materials optical properties. ${ }^{33}$

Recently, we presented and discussed a geometrical model which allows the ellipsometric determination of magnetron-sputtered BN thin-film IR- and VIS-optical constants. We also obtained the average grain $c$-axis orientation within our h-BN thin films and the amount of $c$-BN within high $c$-BN content thin films. ${ }^{25,28-30}$ Visible-light SE spectra of thin films of the wide-gap material BN reveal opticalthickness interference only. On the other hand, IR-SE spectra 
are dominated by the in-plane $(\perp)$, out-of-plane $(\|)$ h-BN, and $c$ - BN $(c)$ transverse $(\mathrm{TO})\left(\omega_{\mathrm{TO}}\right)$ and longitudinal $\left(\omega_{\mathrm{LO}}\right)$ optical (LO) lattice resonance vibrations $\left(\omega_{\mathrm{TO} \|}=783 \mathrm{~cm}^{-1}\right.$, $\omega_{\mathrm{LO} \|}=828 \mathrm{~cm}^{-1}, \quad \omega_{\mathrm{TO} \perp}=1367 \mathrm{~cm}^{-1}, \quad \omega_{\mathrm{LO} \perp}=1610 \mathrm{~cm}^{-1}$, $\left.\omega_{\mathrm{TO} c}=1065 \mathrm{~cm}^{-1}, \quad \omega_{\mathrm{LO} c}=1340 \mathrm{~cm}^{-1}\right) .{ }^{13,14}$ For our polycrystalline h-BN thin films it was demonstrated by Schubert et $a .^{29}$ that the apparent h-BN out-of-plane (in-plane) thinfilm LO resonance vibration $\widetilde{\omega}_{\mathrm{LO} \|}\left(\widetilde{\omega}_{\mathrm{LO} \perp}\right)$ shifts from $\omega_{\mathrm{LO} \|}\left(\omega_{\mathrm{TO} \perp}\right)$ to $\omega_{\mathrm{TO} \|}\left(\omega_{\mathrm{LO} \perp}\right)$ when the average grain $c$-axis orientation angle $\Theta$ changes from perpendicular to parallel to the sample surface, respectively (Figs. 1 and 2 in Ref. 29). The $\widetilde{\omega}_{\mathrm{LO} \|}\left(\widetilde{\omega}_{\mathrm{LO} \perp}\right)$ value depends on the grain's $c$-axis orientation. Thus randomly oriented h-BN material at the $c-\mathrm{BN}$ grain boundaries and the highly oriented h-BN nucleation layer within high $c$-BN content thin films can be distinguished by analysis of the IR-SE data. ${ }^{30}$

Annealing effects due to the escape of moisture from thin-film micropores should be observable by in situ IR- and VIS-SE investigations. Evaporation of water (VIS refractive index $n_{\mathrm{H}_{2} \mathrm{O}}=1.37$ ) from thin films dilutes their effective refractive indices which results in an increase of the FabryPérot interference periods in the VIS-SE spectra. On the other hand, the BN thin-film lattice resonance behavior is modified by the water absorption band at $\omega_{\mathrm{H}_{2} \mathrm{O}}=1640$ $\mathrm{cm}^{-1},{ }^{34}$ which particularly interferes with the h-BN in-plane LO resonance $\widetilde{\omega}_{\mathrm{LO} \perp}$.

In this article we focus on in situ VIS-SE investigations of $c$-BN and h-BN thin-film annealing effects on as-grown samples heated up to $600{ }^{\circ} \mathrm{C}$ under ultrahigh vacuum (UHV) conditions. $^{35}$ The nature of $\mathrm{BN}$ hydrolysis is reversible. Hence, the content of water $\left(\mathrm{H}_{2} \mathrm{O}\right)$ in $\mathrm{BN}$ thin films can be investigated by sample annealing in vacuum or in dry nitrogen $\left(\mathrm{N}_{2}\right)$, and by observation of the reincorporation of $\mathrm{H}_{2} \mathrm{O}$ after sample exposure to atmosphere. From analysis of the ellipsometric data we confirm moisture incorporation in our h-BN sample only, which we explain by h-BN thin-film microporosity. We use an effective-medium approach in combination with our previously presented geometrical model of mixed-phase $\mathrm{BN}$ thin films to estimate the occupied $\left(\mathrm{H}_{2} \mathrm{O}\right)$ and unoccupied (void) fractions in the h-BN thin films. Ex situ VIS-generalized variable angle spectroscopic ellipsometry (gVASE) before and after sample annealing is used to confirm the reversible character of the moisture incorporation process. No changes of the h-BN grain orientations are observed. ${ }^{25}$ Comparison of experimental and generated in situ IR-SE data of the h-BN samples supports our hypothesis of water absorption into h-BN thin-film micropores. No measurable effect of the temperature dependence of either intrinsic h- or $c$-BN optical constants is observable.

\section{EXPERIMENT}

Several BN thin-film samples were prepared by magnetron sputtering onto [100] silicon wafers at different substrate bias voltages, resulting either in pure h-BN or mixedphase high $c$-BN content thin films, as described elsewhere. $^{3,29}$

In situ VIS-SE measurements from 1.5 to $3.5 \mathrm{eV}$ were performed in an UHV chamber with a typical base pressure of $1 \times 10^{-9}$ Torr. A spectroscopic rotating-analyzer ellipsometer was mounted on the chamber with optical access at about $75^{\circ}$ angle-of-incidence $\left(\Phi_{a}\right)$ through birefringencefree fused-quartz windows. The samples were mounted in an upright position. An incidence-angle fine-calibration measurement was performed on a bulklike GaAs sample at room temperature and yielded $\Phi_{a}=74.2^{\circ}$. A detailed description of the experimental setup can be found in Refs. 3 and 5 .

The samples were fixed on a resistor-heater plate for temperature control, and attached to a manipulator for in situ sample rotation and translation. The sample temperature was measured by a thermocouple attached to the sample surface. The h-BN and $c$-BN samples were measured in situ at temperatures of $20^{\circ} \mathrm{C}$ (room temperature), 100,200 , and $300{ }^{\circ} \mathrm{C}$. Then the resistor control was disabled to quench the samples to room temperature (RT). Afterwards the resistor control was set to heat the samples up to $600{ }^{\circ} \mathrm{C}$. After annealing for several hours, and followed by data acquisition, the resistor control was again disabled to quench the samples back to RT. Then the samples were measured at time intervals of several hours keeping the samples in UHV conditions. Additionally, the samples were investigated by multiple angleof-incidence VIS-SE and gVASE in atmosphere before and after annealing, i.e., before mounting the samples into and after removing them from the chamber.

A rotating-polarizer, rotating-compensator Fouriertransform based spectroscopic IR ellipsometer was employed for in situ measurements in the spectral range from 700 to $2000 \mathrm{~cm}^{-1}$. A special small-sized normal-pressure chamber with optical access at $65^{\circ}$ angle-of-incidence and birefringence-free IR windows was used for the in situ IR-SE measurements. The chamber was purged with dry $\mathrm{N}_{2}$ to ensure (i) the absence of atmospheric moisture during data acquisition and (ii) the fast evacuation of moisture evaporating from the sample. For measurements at temperatures above the $\mathrm{H}_{2} \mathrm{O}$ boiling point $\left(\sim 125^{\circ} \mathrm{C}\right)$ the samples were mounted horizontally in contact with the resistor-heater plate and a thermosensing element.

\section{RESULTS AND DISCUSSIONS}

\section{A. In situ VIS-SE investigations}

Figure 1 shows h-BN experimental (symbols) and generated (solid lines) $\Psi$ and $\Delta$ spectra at RT in UHV conditions obtained before (squares) and after annealing at different temperatures (sample h-BN3 in Ref. 28). A conspicuous decrease of the interference periods in $\Psi$ and $\Delta$ after sample annealing is obvious. (Note the change of the distance between the minima in $\Psi$ in, e.g., spectra A and $\mathrm{C}$ on the lowand high-energy sides of the view graph.) The RT "blueshift' ' in $\Psi$ and $\Delta$ after annealing the sample at 300 (triangles) and $600{ }^{\circ} \mathrm{C}$ (in verted triangles) can only be explained as being due to the decrease of the thin-film effective refractive indices. Small thin-film micropores that originated from the deposition process absorb atmospheric moisture after sample exposure to atmosphere. During annealing the incorporated moisture gradually evaporates leaving unoccupied micropores. The remaining thin film appears as an 


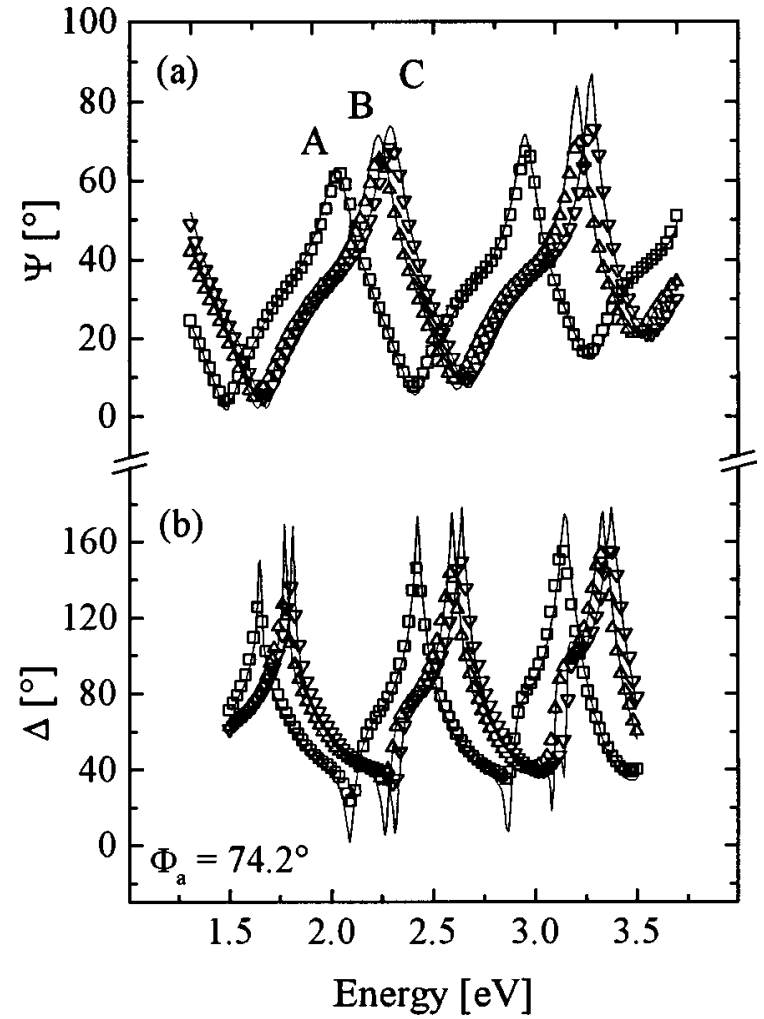

FIG. 1. In situ (UHV, $p=10^{-9}$ Torr) experimental (symbols) and generated (solid lines) VIS-SE $\Psi$ (a) and $\Delta$ (b) RT spectra obtained from our h-BN thin film $\left(\Theta=90^{\circ}\right.$, sample h-BN 3 in Ref. 28). The angle-of-incidence is $\Phi_{a}=74.2^{\circ}$. Measurements were taken before annealing (squares, model-fit A), after annealing for $2 \mathrm{~h}$ at $300{ }^{\circ} \mathrm{C}$ (triangles, B), and after additional annealing for $2 \mathrm{~h}$ at $600{ }^{\circ} \mathrm{C}$ (inverted triangles, C). The increase of the interference periods in both the $\Psi$ and $\Delta$ spectra is due to the decrease of the thin-film effective refractive indices caused by moisture evaporation from the thin-film micro pores [increase of the unoccupied (void) thin-film fraction]. The generated data were taken from the best simultaneous fit for the anisotropic n-BN optical constants $n_{o}$, and $n_{e}$, the film thickness $d$, the h-BN $\left(f_{\mathrm{n}-\mathrm{BN}}=0.65\right)$, moisture $\left(f_{\mathrm{H}_{2} \mathrm{O}}\right)$, and void $\left(f_{\text {void }}\right)$ fractions $\left(\mathrm{A}: f_{\mathrm{H}_{2} \mathrm{O}}\right.$ $=0.35, f_{\text {void }}=0$; B: $\left.f_{\mathrm{H}_{2} \mathrm{O}}=0.07, f_{\text {void }}=0.28 ; \mathrm{C}: f_{\mathrm{H}_{2} \mathrm{O}}=0, f_{\text {void }}=0.35\right)$, see also Table I and Fig. 5.

optically thinner medium. This moisture evaporation and incorporation were found to be reversible. A few days after the annealed h-BN sample was exposed to atmosphere, the blueshift in the $\Psi$ and $\Delta$ spectra disappeared. The VIS-SE data were then identical to data obtained before annealing.

The altering of h-BN thin films by moisture incorporation on exposure to atmosphere was reported by Rand and Roberts. ${ }^{36}$ The authors observed an additional broad absorption band at $3300 \mathrm{~cm}^{-1}$ in the IR spectra after the sample was stored in room ambient for a few weeks. An additional IR band in BN thin-film spectra at $3300 \mathrm{~cm}^{-1}$ was also reported by Hahn et al. ${ }^{3}$ and by Cardinale et al. ${ }^{37}$ They suggest a possible reaction between $\mathrm{h}-\mathrm{BN}$ and moisture by $\frac{13}{2} \mathrm{O}_{2}+8 \mathrm{BN}+\mathrm{H}_{2} \mathrm{O} \rightarrow 2\left[\left(\mathrm{~B}_{2} \mathrm{O}_{3}\right)_{2}(\mathrm{OH})\right]+4 \mathrm{~N}_{2}$. Hubácek and $\mathrm{Ueki}^{8}$ explained the moisture-incorporation process by $\mathrm{BN}$ hydrolysis, where hydroxyl $(\mathrm{OH})$ and hydrogen are bonded by nitrogen and boron at the boundaries of the hexagonal BN network. Furthermore, molecular water may be absorbed by thin-film micropores. Because of the reversible character of the moisture incorporation our h-BN thin films, the latter

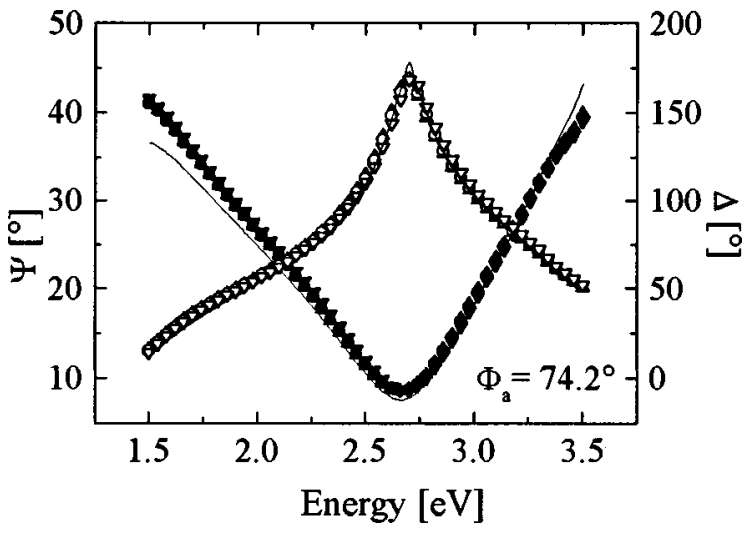

FIG. 2. In situ (UHV) experimental (symbols) and generated (solid lines) VIS-SE $\Psi$ (closed symbols) and $\Delta$ (open symbles) RT spectra obtained for our $c$-BN thin film ( $c$-BN2 in Ref. $28 ; \Phi_{a}=74.2^{\circ}$; triangles: before annealing, circles: after annealing at $300{ }^{\circ} \mathrm{C}$, inverted triangles: after annealing at $600{ }^{\circ} \mathrm{C}$. No shift in $\Psi$ and $\Delta$ is observable upon sample annealing. The latter is expected as the high $c$-BN content films are more dense than the h-BN thin films. The generated data are obtained using the isotropic optical constant $n_{c}$ and the thin-film thickness $d$ abstracted from Ref. 28 .

process is most likely dominant. Transmission electron microscopy (TEM) investigations of our h-BN thin films revealed a microporous sample structure, supporting the assumption of molecular water absorption therein.

Figure 2 shows in situ RT experimental VIS-SE $\Psi$ (closed symbols) and $\Delta$ (open symbols) spectra of a high $c$-BN content thin-film sample ( $c$-BN2 in Ref. 28) before and after annealing in $\operatorname{UHV}\left(\Phi_{a}=74.2^{\circ}\right)$. The RT spectra, taken after several hours of annealing at different temperatures, are identical, and no changes of the $c$-BN thin-film VIS optical properties are observable after the annealing process. Obviously, no moisture exchange, as was observed for the h-BN sample, occurs. Because of higher thin-film densities obtained by the particular $c$-BN growth conditions, water diffusion and incorporation processes in the as-grown samples do not occur.

Figures 3(a) and 3(b) present in situ VIS-SE $\Psi$ and $\Delta$ spectra, respectively, of the h-BN thin film at $T=600^{\circ} \mathrm{C}$ (squares) and at RT (diamonds) after annealing in UHV at $T=600{ }^{\circ} \mathrm{C}$ for several hours $\left(\Phi_{a}=74.2^{\circ}\right)$. The spectra taken at different sample temperatures are now nearly identical. We conclude that after evaporation of the thin-film moisture incorporation, the remaining h-BN thin-film refractive indices are almost temperature independent. A flat temperature dependence of the h-BN dielectric properties was reported by Zunger et al. ${ }^{38}$ and is confirmed by our thin-film experiment.

In situ VIS-SE $\Psi$ (closed symbols) and $\Delta$ (open symbols) spectra of the high $c$-BN content thin film at $T$ $=600{ }^{\circ} \mathrm{C}$ and at $\mathrm{RT}$ after annealing at $T=600{ }^{\circ} \mathrm{C}$ in UHV are shown in Fig. $4\left(\Phi_{a}=74.2^{\circ}\right)$. Similar to the h-BN sample, no change of the $c$-BN thin-film refractive indices at elevated temperatures with respect to RT is observable. We conclude that RT refractive indices of both $\mathrm{h}$ - and $c$-BN can be used for real-time analysis of in situ SE data, regardless of the sample temperature, until at least $T=600^{\circ} \mathrm{C}$. It is worth noting that SE data are very sensitive to critical-point contri- 


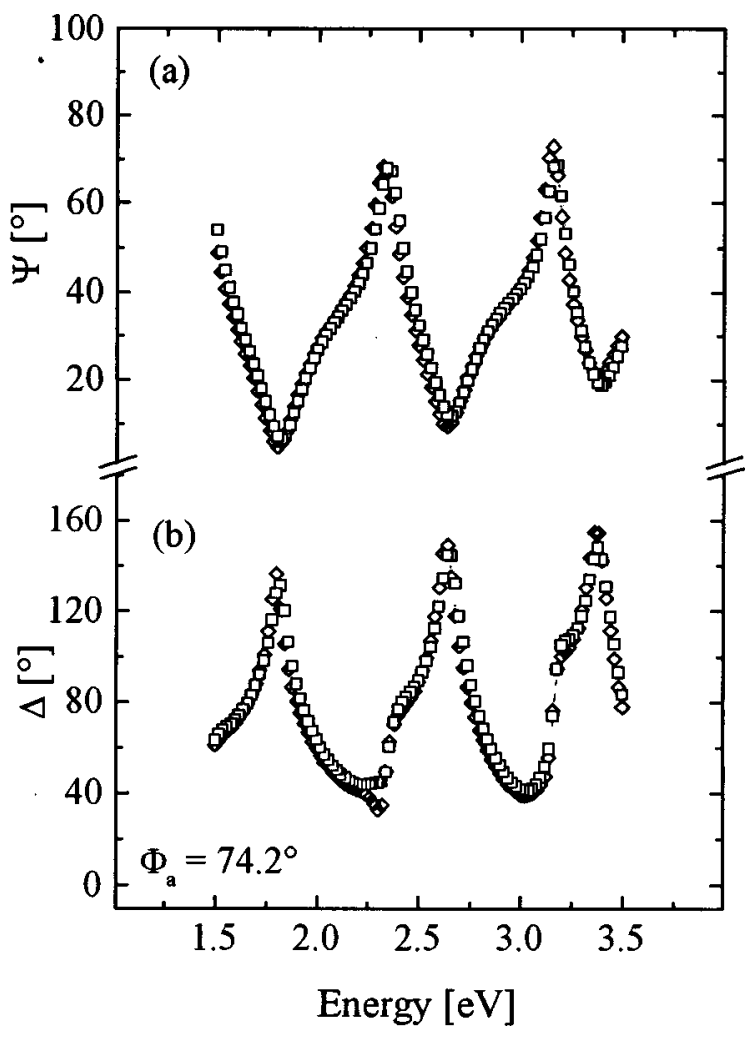

FIG. 3. In situ (UHV) experimental VIS-SE $\Psi$ (a) and $\Delta$ (b) spectra obtained from the h-BN sample at $600{ }^{\circ} \mathrm{C}$ after annealing for several hours (squares) and at RT (diamonds) after annealing at $600^{\circ} \mathrm{C}\left(\Phi_{a}=74.2^{\circ}\right)$. No measurable shift in $\Psi$ and $\Delta$, and hence no temperature dependence of the h-BN thin-film refractive indices are observed.

butions of III-V compound materials. ${ }^{39,40}$ Such critical-point structures are known to be temperature dependent. Dependencies like this can be used for precise in situ sampletemperature control. ${ }^{41}$ However, due to the large band gap of $\mathrm{BN}\left[E_{g}(c-\mathrm{BN}) \sim 6.1 \mathrm{eV},{ }^{42} E_{g}(\mathrm{~h}-\mathrm{BN}) \sim 5.5-5.9 \mathrm{eV}\right]^{43}$ even the lowest critical-point structure of $\mathrm{BN}$ was inaccessible for our in situ spectroscopic setup, which was limited to $5 \mathrm{eV}$.

We use an effective-medium approach to analyze the VIS-SE data of the h-BN sample. ${ }^{44}$ As discussed earlier, our

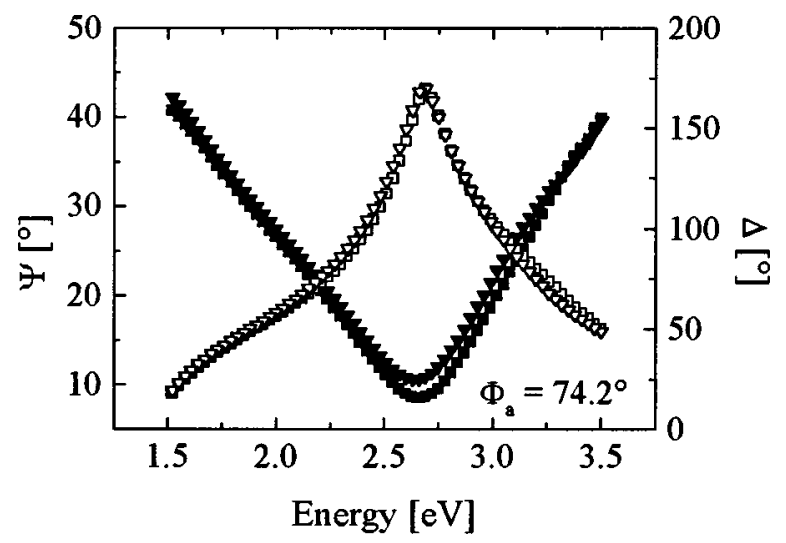

FIG. 4. In situ (UHV) experimental VIS-SE $\Psi$ (closed symbols) and $\Delta$ (open symbols) spectra obtained from the $c$-BN thin film at RT (squares) and at $600{ }^{\circ} \mathrm{C}$ (diamonds, $\Phi_{a}=74.2^{\circ}$ ). This is similar to the annealed h-BN sample; no appreciable temperature dependence of the $c$-BN optical constants is observable.
h-BN samples possess uniaxial birefringence with optical axis perpendicular to the thin-film surface. This birefringence arises from the coned arrangement with angle-ofspread $\Theta$ of the uniaxial h-BN polycrystals. ${ }^{25}$ In order to account for different moisture and void (empty micropores) fractions we assume a linear law to average the effective thin-film h-BN dielectric function tensor as follows:

$$
\tilde{\epsilon}_{\mathrm{h}, \mathrm{eff}}=f_{\mathrm{h}-\mathrm{BN}} \epsilon_{\mathrm{h}-\mathrm{BN}}+f_{\mathrm{H}_{2} \mathrm{O}} \epsilon_{\mathrm{H}_{2} \mathrm{O}}+f_{\text {void }} \epsilon_{\mathrm{void}},
$$

with

$$
\begin{aligned}
& \epsilon_{\mathrm{h}-\mathrm{BN}}=\operatorname{diag}\left(n_{o, \text { eff }}^{2}, n_{o, \text { eff }}^{2}, n_{e, \text { eff }}^{2}\right), \\
& \epsilon_{\mathrm{H}_{2} \mathrm{O}}=\operatorname{diag}\left(n_{\mathrm{H}_{2} \mathrm{O}}^{2}, n_{\mathrm{H}_{2} \mathrm{O}}^{2}, n_{\mathrm{H}_{2} \mathrm{O}}^{2}\right), \\
& \epsilon_{\mathrm{void}}=\operatorname{diag}(1,1,1),
\end{aligned}
$$

and

$$
\begin{aligned}
& n_{o, \mathrm{eff}}^{2}=0.25\left[(3+\cos 2 \Theta) n_{o}^{2}+(1-\cos 2 \Theta) n_{e}^{2}\right], \\
& n_{e, \mathrm{eff}}^{2}=\left(n_{e} \cos \Theta\right)^{2}+\left(n_{o} \sin \Theta\right)^{2},
\end{aligned}
$$

where $n_{o}, n_{e}, n_{\mathrm{H}_{2} \mathrm{O}}$ are the ordinary (perpendicular $c$ ), extraordinary n-BN, and $\mathrm{H}_{2} \mathrm{O}$ refractive indices, respectively, and $\operatorname{diag}(\mathrm{)})$ indicates the diagonal matrix. The h-BN, $\mathrm{H}_{2} \mathrm{O}$, and void fraction parameters $f_{\mathrm{h}-\mathrm{BN}}, f_{\mathrm{H}_{2} \mathrm{O}}$, and $f_{\text {void }}$ are used to estimate the respective thin-film content. It follows that $f_{\mathrm{h}-\mathrm{BN}}+f_{\mathrm{H}_{2} \mathrm{O}}+f_{\text {void }}=1$. The intrinsic $\mathrm{h}-\mathrm{BN}$ refractive indices follow the usual Cauchy dispersion law, and are modeled by three coefficients $\alpha, \beta$, and $\gamma$ :

$$
n_{o, e}=\alpha_{o, e}+\frac{\beta_{o, e}}{\lambda^{2}}+\frac{\gamma_{o, e}}{\lambda^{4}},
$$

with $\lambda$ being the vacuum wavelength of the incident light (given in units of eV through Figs. 1-4). Figure 1 shows generated data (solid lines) using the results of the best fit for $n_{o}, n_{e}\left(\alpha_{o, e}, \beta_{o, e}, \gamma_{o, e}\right), f_{\mathrm{h}-\mathrm{BN}}, f_{\mathrm{H}_{2} \mathrm{O}}, f_{\text {void }}$, and thickness $d$ to the experimental data. Note that a pseudomultiple-sample best fit was employed for the regression analysis. Multiplesample analysis is known to provide sensitivity to the sample structure when both the film thickness and the film optical constants are unknown. ${ }^{45,46}$ In particular, the spectra labeled by letters $\mathrm{A}, \mathrm{B}$, and $\mathrm{C}$ in Fig. 1 refer to data taken on the same sample, and at the same position after different temperature ramping and cooling. For analysis, the same thickness $d$, h-BN content $f_{\mathrm{h}-\mathrm{BN}}$, and angle-of-spread $\Theta$ (i.e., the same microstructure of the h-BN matrix) were assumed. The validity of the latter assumption is evidenced through the IR results, and will be discussed in Sec. III B. The volume fraction parameters are now correlated in that we have $f_{\mathrm{h}-\mathrm{BN}}$ $=1-f_{\mathrm{H}_{2} \mathrm{O}}-f_{\text {void }}$ or $f_{\mathrm{H}_{2} \mathrm{O}}+f_{\text {void }}=$ const for all data sets.

The results of the regression analysis for the h-BN sample are given in Table I. We estimate from our calculation that only $65 \%$ of the volume content of the thin film consists of polycrystalline h-BN material. The remaining $35 \%$ are empty micropores which are partially filled by water. As the sample is treated at different temperatures, moisture evaporates gradually until saturation of the void content (only empty micropores remaining). Hence, the spectra labeled $\mathrm{A}, \mathrm{B}$, and $\mathrm{C}$ can be modeled by (h- $\mathrm{BN}, \mathrm{H}_{2} \mathrm{O}$, void) 
TABLE I. VIS-SE sample-structure parameter results.

\begin{tabular}{lccccccccc}
\hline \hline Sample & $\begin{array}{c}d \\
(\mathrm{~nm})\end{array}$ & $E$ & $\alpha$ & $\left(10^{-3} \mu \mathrm{m}^{-2}\right)$ & $\left(10^{-3} \mu \mathrm{m}^{-4}\right)$ & $\begin{array}{c}\text { Data } \\
\text { set }\end{array}$ & $f_{\mathrm{n}-\mathrm{BN}}$ & $f_{\mathrm{H}_{2} \mathrm{O}}$ & $f_{\text {void }}$ \\
\hline $\mathrm{h}-\mathrm{BN}^{\mathrm{a}}$ & & $\perp c$ & 1.97 & 0.6 & 1.9 & $\mathrm{~A}$ & 0.65 & 0.35 & 0 \\
& 531 & $\| c$ & 1.59 & 3.1 & 0.9 & $\mathrm{~B}$ & 0.65 & 0.07 & 0.28 \\
& & & & & & $\mathrm{C}$ & 0.65 & 0 & 0.35 \\
\hline \hline
\end{tabular}

${ }^{\mathrm{a}}$ Sample h-BN3 in Ref. 28.

fractions $\left(f_{\mathrm{h}-\mathrm{BN}}, f_{\mathrm{H}_{2} \mathrm{O}}, f_{\text {void }}\right)$ as $(0.65,0.35,0),(0.65,0.07$, $0.28)$, and $(0.65,0,0.35)$ only, respectively. The intrinsic h-BN refractive indices $n_{o}$ (closed circles), and $n_{e}$ (closed squares) are shown in Fig. 5 together with BN optical constants presented recently (open symbols) in the literature. ${ }^{25,28}$ In these previous papers we did not account for the moisture incorporation which lowers the apparent thin-film refractive indices. Consequently, $n_{o}$ and $n_{e}$ obtained in this work are considerably higher than in Ref. 25 (see also Figs. 8 and 9 in Ref. 47.) However, ordinary and extraordinary h-BN refractive indices reported for bulk material $\left[n_{e}=1.65, n_{o}=2.13\right.$ (Ref 48)] still exceed our findings.

The generated data shown in Fig. 2 for our $c$-BN sample are from Ref. 28 (sample $c$-BN2 in Fig. 3(b) and Table I). The in situ VIS-SE data reported here are identical to the $e x$ situ VIS-SE data obtained in Ref. 28. Note that we used the Bruggeman effective-medium approximation ${ }^{44}$ to consistently analyze both the IR- and the VIS-SE data reported in Ref. 28. In particular, we have calculated the amount of h-BN within the high $c$-BN. content sample. We have, as well, determined the orientation and optical thickness of the $s p^{2}$-bonded nucleation layer. Therefore, we omit further discussion of the $c$-BN sample investigated here, and refer the reader to Ref. 28 and references therein. The $c$-BN VIS refractive index $n_{c}$ is presented in Fig. 5 for comparison.

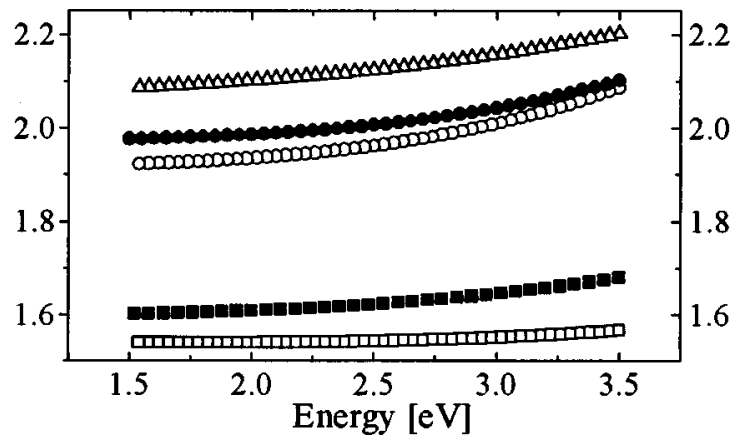

FIG. 5. BN refractive indices obtained from the multiple-sample analysis presented in Ref. 25 without consideration of moisture incorporation in the h-BN samples ( $n_{c}$ for $c$-BN: open triangles, $n_{e}$ for h-BN: open squares, $n_{o}$ for h-BN: open circles). The closed symbols show the h-BN refractive indices obtained in this work. These values are higher than the those previously obtained (open symbols), since in Ref. 25 we did not account for the low-refractive index incorporation which lower the apparent h-BN thin-film refractive indices. However, the values presented here are still below the h-BN refractive indices reported for bulk material $\left[n_{o}=1.65, n_{e}=2.13\right.$ (Ref. 48)].

\section{B. In situ IR-SE investigations}

As is discussed in Ref. 28, the IR optical properties of h-BN are modeled by describing each intrinsic lattice resonance absorption as a Lorentz oscillator [see Eq. (3) in Ref. 29 and see also Geick, Perry, and Rupprecht, Ref. 13]. The h-BN thin-film dielectric function (DF) tensor properties are formally identical to Eq. (1), where $n_{e, \text { eff }}^{2}=\epsilon_{e, \text { eff }}$ and $n_{o \text {,eff }}^{2}$ $=\epsilon_{o \text { eff }}$ are for DFs for polarization parallel and perpendicular to the film normal [see Eqs. 2(a) and 2(b) in Ref. 29]. Figure 6 shows generated (simulated) IR-SE $\Psi$ and $\Delta$ spectra $\left(\Phi_{a}\right.$

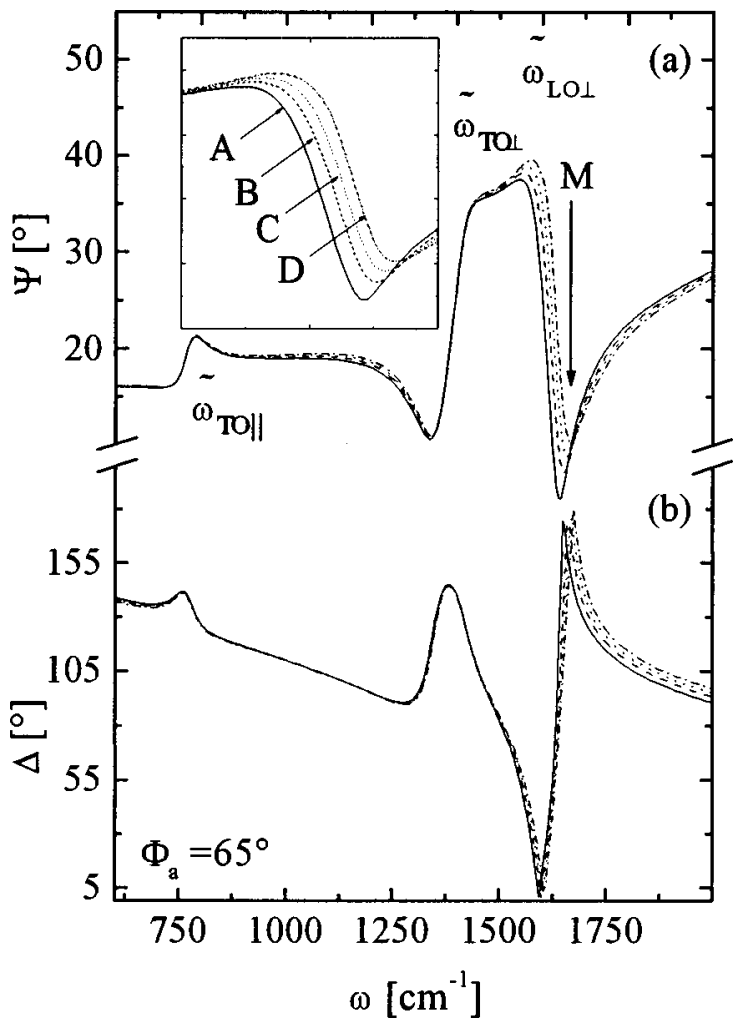

FIG. 6. Generated (simulation) IR-SE $\Psi$ (a) and $\Delta$ (b) spectra $\left(\Phi_{a}=65^{\circ}\right)$ for an in-plane oriented $\left(\Theta=90^{\circ}\right)$ microporous $\left(f_{h-B N}=0.65\right)$ polycrystalline h-BN thin film $(d=550 \mathrm{~nm})$ with different (moisture, void) fractions $\left(f_{\mathrm{H}_{2} \mathrm{O}}, f_{\text {void }}\right)$. A: $(0.35,0)$ solid line, B: $(0.20,0.15)$ dashed line, C: $(0.10$, $0.25)$ dotted line, D: $(0,0.35)$ dash-dotted line). The remaining parameters are taken from Table I in Ref. 29 (sample hBN2). While the h-BN out-ofplane lattice resonance behavior does not show any moisture-content dependencies, the apparent in-plane LO resonance frequency $\widetilde{\omega}_{\mathrm{LO} \perp}$ becomes more pronounced and shifts to higher wave numbers as the unoccupied thin-film content increases. Note also the spectral shift of the minimum $(M)$ in $\Psi$ above $\widetilde{\Psi}_{\mathrm{LO} \perp}$ which refers to the minimum in $R_{p}$ above the thin-film longitudinal optical lattice resonance. 
$\left.=65^{\circ}\right)$ for an in-plane oriented $\left(\Theta=90^{\circ}\right)$ microporous $\left(f_{\mathrm{h}-\mathrm{BN}}=0.65\right)$ polycrystalline h-BN thin film $(d=550 \mathrm{~nm})$ for different (moisture, void) fractions $\left(f_{\mathrm{H}_{2} \mathrm{O}}, f_{\text {void }}\right)$. The h-BN lattice resonance parameters used here are taken from sample hBN2 (see Table I in Ref. 29; sample hBN2 there was relabeled as hBN3 in Ref. 28). Tabulated IR optical constants for $\mathrm{H}_{2} \mathrm{O}$ are given in Ref. 49. Obviously, only the in-plane lattice resonance behavior of the h-BN thin film is influenced by moisture incorporation. Because of the water absorption band at $\omega_{\mathrm{H}_{2} \mathrm{O}}=1640 \mathrm{~cm}^{-1}$ (Ref. 34) the apparent h-BN mode $\widetilde{\omega}_{\mathrm{LO} \perp}$ shifts to higher wave numbers as the water content decreases (see the inset in Fig. 6). In addition, the extrema associated with $\widetilde{\omega}_{\mathrm{LO} \perp}$ become more pronounced. On the other hand, the out-of-plane optical behavior remains almost unchanged. In Ref. 29 we thoroughly discussed the IR optical properties of BN thin films as a function of their microstructure. We demonstrated that changes of the microstructure affect both in- and out-of-plane lattice resonance spectral regions. In particular, we showed the existence of spectral shifts of both longitudinal optical thin-film vibrations. Therefore, if the thin film would change its microstructure during annealing, one would expect to observe changes in both in- and out-of-plane spectral regions.

Figure 7 presents experimental in situ IR-SE $\Psi$ and $\Delta$ spectra of our h-BN sample before and during annealing in dry $\mathrm{N}_{2}$ at $T=132^{\circ} \mathrm{C}$ (A: squares, in atmosphere at RT, B: circles, in dry $\mathrm{N}_{2}$ at $132^{\circ}$ after $4 \mathrm{~h}$ annealing time, C: triangles, in dry $\mathrm{N}_{2}$ at RT after $18 \mathrm{~h}$ annealing time). The $\Psi$ and $\Delta$ spectra show the same behavior as shown in Fig. 6. The apparent $\widetilde{\omega}_{\mathrm{LO} \perp}$ resonance frequency shifts to higher wave numbers after $4 \mathrm{~h}$ of annealing. Also, the extrema corresponding to $\widetilde{\omega}_{\mathrm{LO} \perp}$ become more pronounced (see the inset in Fig. 7). No further shift is observable upon extending the annealing time. Note that due to the specific construction of the temperature-control setup, this experiment was limited to $\sim 135{ }^{\circ} \mathrm{C}$. The data obtained at RT (in dry $\mathrm{N}_{2}$ ) after $18 \mathrm{~h}$ annealing are identical with those obtained at $132{ }^{\circ} \mathrm{C}$. Hence, the observed changes are not due to temperature effects. Again, after some days of sample exposure to atmosphere, the IR-SE data are identical (not shown here) to data set A (i.e., before annealing) as presented in Fig. 6. We conclude that moisture evaporation and reincorporation cause the changes of the IR-SE data reported here, in accordance with the experimental observations in the VIS spectral range as discussed in Sec. III A.

\section{CONCLUSIONS}

We have presented in situ VIS- and IR-SE investigations at RT and at elevated temperatures performed on h-BN and $c$-BN thin films. We observe a reversible blueshift in the h-BN VIS-SE data upon annealing and upon sample reexposure to atmosphere. No annealing effects are measured for the $c$-BN sample. The changes of the h-BN thin-film VIS refractive indices can be explained by the thin-film void fraction partially absorbed by water. When annealed in UHV or in dry nitrogen, the h-BN sample releases moisture but retains its microstructure. Incorporation of moisture by the thin-film micropores affects the IR in-plane lattice resonance

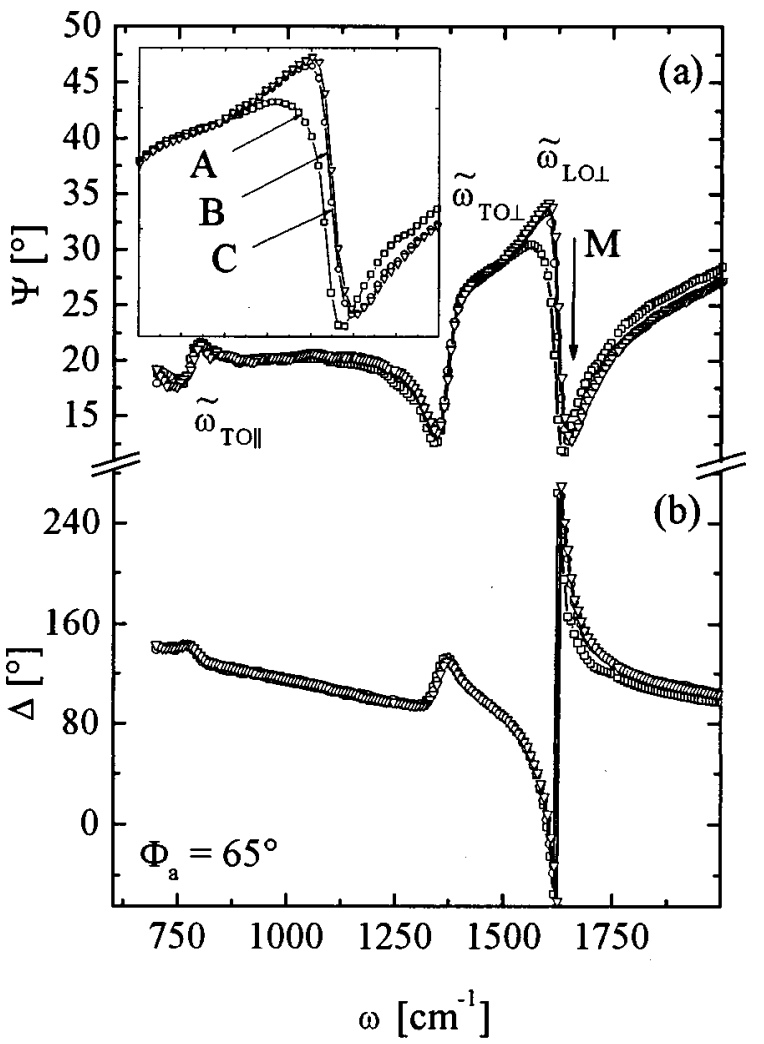

FIG. 7. In situ IR-SE $\Psi$ (a) and $\Delta$ (b) spectra from our h-BN thin film $\left(\Phi_{a}=65^{\circ}\right)$. Measurements were taken before annealing, in atmosphere at RT (A, squares), at $132{ }^{\circ} \mathrm{C}$ in dry $\mathrm{N}_{2}$ after $4 \mathrm{~h}$ annealing time (B, circles), and $18 \mathrm{~h}$ annealing time at RT in dry $\mathrm{N}_{2}$ (C, inverted triangles). The $\Psi$ and $\Delta$ spectra show the same behavior as discussed and shown in Fig. 6. While the extreme which correspond to $\widetilde{\omega}_{\mathrm{LO} \perp}$ and loss of reflectivity above the thin-film longitudinal lattice resonance $(M)$ shift to higher wave numbers, the out-of-plane lattice resonance behavior is not influenced as the annealing process proceeds and the moisture content decreases. The changes in $\Psi$ and $\Delta$ within the in-plane resonance spectral range are due to the moisture evaporation from the thin-film micropores and an increase of the thin-film void fraction. This process is reversible. The data taken before annealing and after sample exposure to atmosphere for some days (not shown here) are almost identical.

behavior which is observed by in situ IR-SE. Transmission electron microscopy images also reveal a porous structure of the sample investigated, and further support our conclusion.

Consideration of the microporous structure of the h-BN sample leads to intrinsic ordinary and extraordinary refractive indices which are higher than those reported recently for similar samples where we did not account for moisture inclusion. However, the bulk optical constants still exceed our results. We further conclude that there is a flat temperature dependence of both h- and $c$-BN VIS optical constants. Therefore, RT refractive indices of BN can be used for in situ feedback control of BN thin-film growth up to temperatures of $600{ }^{\circ} \mathrm{C}$.

\section{ACKNOWLEDGMENTS}

The authors wish to acknowledge G. Wagner for TEM investigations. They further thank Professor F. Richter and Professor F. Bigl for stimulating discussions. The authors express their gratitude to Mick DeVries for assistance during 
the infrared ellipsometry experiments, and thank the Center for Microelectronic and Optical Materials Research of the University of Nebraska-Lincoln for financial support during part of the work. One of the authors (M.S.) acknowledges financial support of the Deutsche Forschungsgemeinschaft, Grant No. Rh28/1-1, and thanks the Studienstiftung des Deutschen Volkes.

${ }^{1}$ J. M. Mendez, S. Muhl, M. Farias, G. Sato, and L. Cota-Araiza, Surf. Coat. Technol. 41, 422 (1991).

${ }^{2}$ S. Reinke, M. Kuhr, W. Kulisch, and R. Kassing, Diamond Relat. Mater. 4, 272 (1995).

${ }^{3}$ J. Hahn, M. Friedrich, R. Pintaske, M. Schaller, N. Kahl, D. R. T. Zahn, and F. Richter, Diamond Relat. Mater. 5, 1103 (1996).

${ }^{4}$ T. A. Friedmann, P. B. Mirkarimi, D. L. Medlin, K. F. McCarty, E. J. Klaus, D. R. Boehme, H. A. Johnson, M. J. Mills, and D. K. Otteson, J. Appl. Phys. 76, 3088 (1994).

${ }^{5}$ N. Tanabe, T. Hayashi, and M. Iwaki, Diamond Relat. Mater. 1, 883 (1992).

${ }^{6}$ H. Hofsäss, C. Ronning, U. Griesmeier, S. Reinke, and M. Kuhr, Appl. Phys. Lett. 67, 46 (1995).

${ }^{7}$ K.-L. Barth, A. Neuffer, J. Ulmer, and A. Lunk, Diamond Relat. Mater. 5, 1270 (1996).

${ }^{8}$ M. Hubácek and M. Ueki, J. Solid State Chem. 123, 215 (1996).

${ }^{9}$ D. H. Berns and M. A. Capelli, J. Mater. Res. 12, 2014 (1997).

${ }^{10}$ M. Okamoato, Y. Utsumi, and Y. Osaka, Jpn. J. Appl. Phys., Part 1 31, 3455 (1992).

${ }^{11}$ M. I. Eremets, M. Gauthier, A. Polian, J. C. Chervin, J. M. Besson, G. A. Dubitskii, and Y. Y. Semenova, Phys. Rev. B 52, 8854 (1995).

${ }^{12}$ A. S. Rozenberg, Y. A. Sinenko, and N. V. Chukanov, J. Mater. Sci. 28, 5675 (1993)

${ }^{13}$ R. Geick, C. H. Perry, and G. Rupprecht, Phys. Rev. 146, 543 (1966).

${ }^{14}$ P. J. Gielisse, S. S. Mitra, J. N. Plendl, R. Griffis, L. C. Mannsur, R. Marshall, and E. A. Pascoe, Phys. Rev. 155, 1039 (1967).

${ }^{15}$ R. J. Nemanich, S. A. Solin, and R. M. Martin, Phys. Rev. B 23, 6348 (1982).

${ }^{16}$ H. Herchen and M. A. Capelli, Phys. Rev. B 47, 14193 (1993).

${ }^{17}$ G. J. Exarhos and J. W. Schaaf, J. Appl. Phys. 69, 2543 (1991).

${ }^{18}$ A. D. Alvarenga, M. Grimsditch, and A. Polian, J. Appl. Phys. 72, 1955 (1992).

${ }^{19}$ T. Kuzuba, K. Era, T. Ishii, and T. Sato, Solid State Commun. 25, 863 (1978).

${ }^{20}$ D. M. Hoffman, G. L. Doll, and P. C. Eklund, Phys. Rev. B 30, 6051 (1984).

${ }^{21}$ S. L. Ren, A. M. Rao, P. C. Eklund, and G. L. Doll, Appl. Phys. Lett. 62, 1760 (1993)

${ }^{22}$ D. R. McKenzie, W. D. McFall, H. Smith, B. Higgins, R. W. Boswell, A.
Durandet, B. W. James, and J. S. Falconer, Nucl. Instrum. Methods Phys. Res. B 106, 90 (1995).

${ }^{23}$ E. Pascual, J. L. Andújar, S. Gimeno, A. Lousa, A. Bosch, M. El Kasmi, and E. Bertran, Diamond Relat. Mater. 5, 539 (1996).

${ }^{24}$ C. Quirós, P. Prieto, J. F. Trigo, E. L. Elizalde, and J. M. Sanz, Nucl. Instrum. Methods Phys. Res. B 112, 275 (1996).

${ }^{25}$ M. Schubert, B. Rheinländer, E. Franke, H. Neumann, J. Hahn, M. Röder, and F. Richter, Appl. Phys. Lett. 70, 1819 (1997).

${ }^{26}$ M. C. Polo, M. Ben el Mekki, J. L. Andújar, N. Mestres, and J. Pascual, Diamond Relat. Mater. 6, 1550 (1997).

${ }^{27}$ M. F. Plass, W. Fukarek, S. Mändl, and W. Möller, Appl. Phys. Lett. 69, 46 (1996).

${ }^{28}$ E. Franke, M. Schubert, H. Neumann, T. E. Tiwald, D. W. Thompson, J. A. Woollam, J. Hahn, and F. Richter, J. Appl. Phys. 82, 2906 (1997).

${ }^{29}$ M. Schubert, B. Rheinländer, E. Franke, H. Neumann, T. E. Tiwald, J. A. Woollam, J. Hahn, and F. Richter, Phys. Rev. B 56, 13306 (1997).

${ }^{30}$ E. Franke, H. Neumann, M. Schubert, T. E. Tiwald, J. A. Woollam, and J. Hahn, Appl. Phys. Lett. 70, 1668 (1997).

${ }^{31}$ R. W. Collins, I. An, H. V. Nguyen, and Y. Lu, Thin Solid Films 233, 244 (1993).

${ }^{32}$ R. M. A. Azzam and N. M. Bashara, Ellipsometry and Polarized Light (North-Holland, Amsterdam, 1977).

${ }^{33}$ G. E. Jellison, Thin Solid Films 234, 416 (1993).

${ }^{34}$ A. Brunet-Bruneau, J. Rivory, B. Rafin, J. Y. Robic, and P. Chaton, J. Appl. Phys. 82, 1330 (1997).

${ }^{35}$ H. Yao, P. G. Snyder, and J. A. Woollam, J. Appl. Phys. 70, 3261 (1991).

${ }^{36}$ M. Rand and J. F. Roberts, J. Electrochem. Soc. 15, 423 (1968)

${ }^{37}$ G. F. Cardinale, P. B. Mirkarimi, K. F. McCarty, E. J. Klaus, D. Medlin, W. M. Clift, and D. G. Howitt, Thin Solid Films 253, 130 (1994).

${ }^{38}$ A. Zunger, A. Katzir, and A. Halperin, Phys. Rev. B 13, 5560 (1976).

${ }^{39}$ P. Lautenschlager, M. Garriga, and M. Cardona, Phys. Rev. B 36, 4813 (1987).

${ }^{40}$ P. Lautenschlager, M. Garriga, L. Viña, and M. Cardona, Phys. Rev. B 36, 4821 (1987).

${ }^{41}$ G. Vuye, S. Fisson, V. N. Nguyen, Y. Wang, J. Rivory, and F. Abelés, Thin Solid Films 233, 166 (1993).

${ }^{42}$ N. Miyata et al., Phys. Rev. B 40, 12028 (1989).

${ }^{43}$ C. A. Taylor, S. W. Brown, V. Subramaniam, S. Kidner, S. C. Rand, and R. Clarke, Appl. Phys. Lett. 65, 1251 (1994).

${ }^{44}$ D. E. Aspnes and J. B. Theeten, Phys. Rev. B 20, 1669 (1979).

${ }^{45}$ W. A. McGahan, B. Johs, and J. A. Woollam, Thin Solid Films 234, 443 (1993)

${ }^{46}$ N. V. Nguyen, D. Chandler-Horowitz, P. M. Amirtharaj, and J. P. Pellegrino, Appl. Phys. Lett. 64, 2688 (1994)

${ }^{47}$ M. Schubert, Thin Solid Films (to be published).

${ }^{48}$ R. Poerschke and O. Madelung, Semiconductor Group IV Elements and III-V Compounds (Springer, Berlin, 1991).

${ }^{49}$ E. D. Palik, Handbook of Optical Constants (Academic, New York, 1991), Vol. II. 